\title{
Sources of valuable characters of pea and chickpea for breeding
}

\section{Krynychna N.}

NSC «Institute of soil science and agrochemistry research named after O. N. So-kolovsky», 61024, Chaikovska Str., 4, Kharkiv; e-mail: n.krinichnaya@ukr.net

The purpose. To add new samples of pease and chick pea to base collection of laboratory that enables to diversify genetic material of these crops. Owing to study and analysis to enrich collection with sources of high productivity and autoadaptivity to conditions of Steppe of Ukraine. Methods. Objects of probes collection samples of pease and chick pea. Sowing was carried out in the optimum for pea and chick pea time with the help of hand planters on plots with area of $1 \mathrm{~m}^{2}$ using $1510 \mathrm{~cm}$ scheme of planting. Block of standards followed each 10 samples. Morphological description of samples, their grading on economic and biological characters was carried out using classifiers of conforming stems. Statistical analysis of the gained results was realized using standard mathematical methods. Results. Results are brought of study of collection samples of pease and chick pea during 2015-2017 in contrasting ambient conditions. That made it possible to determine selection- and economic-valuable material. Samples with stable during years level of development of attributes are specified as approved samples of yielding ability, productivity, mass of 1000 grains, height of plants and height of lower raceme, etc. These approved samples can be used in selection work. Conclusions. As a result of the conducted analysis samples of pease and chick pea which have high technological and productive parameters are determined. Pease: LNAU I/13, LDS01180, UKR which has high technological and productive parameters (middle ripening, tall, suitable to mechanical harvesting, mass of 1000 seeds - 215,5 g, productivity - 283,3 g/ $\mathrm{m}^{2}$ ); Lug 76/13, LDS01168, UKR (middle ripening, suitable to mechanical harvesting, high productivity - 318,9 g/ $\mathrm{m}^{2}$ ); Lug 118/13, LDS01186, UKR (middle ripening, suitable to mechanical harvesting, productivity $-284,4 \mathrm{~g} / \mathrm{m}^{2}$ ). Samples are stable against diseases and pests. Chick pea: Odissei, LDS00810, UKR; Skarb, LDS00811, UKR; Lug 89/10, LDS00827, UKR; Lug 99/11, LDS00826, UKR; Lug 101/11, LDS00828, UKR. All these samples are early, suitable for mechanical harvesting, have very large seeds $(>350 \mathrm{~g})$, secure heavy yield of seeds, have stagnant shape of a scrub. All samples of pease and chick pea had big diversification of attributes at their growing in zone of Steppe of Ukraine. At creation of material with hi-tech and efficient parameters it is possible to recommend all the samples indicated above which combine valuable economic attributes, i.e. samples on complex of attributes.

Key words: pease, chick pea, donor, genetic resources of plants, study.

$$
\text { https://doi.org/10.31073/agrovisnyk201901-07 }
$$

The genetic diversity of species, varieties and forms of plants, which differ in the direction of use, product quality, adaptability, and other valuable economic characteristics are one of the main factors in ensuring food security and continuous development of agricultural production. The need to collect and preserve samples of the plant gene pool for present and future generations is also due to the fact that due to the rapid development of scientific and technological progress, environmental problems, many wild species disappear, the variety of cultivated plants narrows. At the same time, thanks to the use of new technologies, the development of genetics, genetic engineering and biotechnology, the value and role of germ plasma is growing significantly. Wild species, ancient local varieties, as well as breeding varieties created by world science over the past hundred years, in their genetic systems carry many valuable genes, their hereditary basis will always be the source of source material for the creation of new generations of varieties.

The purpose of our work was to attract new samples of peas and chickpea to the basic collection of the laboratory, which allows to diversify the genetic material of these crops, through the study and 
analysis to enrich the collection of sources of high yield and adaptability to the conditions of the Steppe zone of Ukraine.

For further effective and purposeful use of gene pool samples, it is necessary to establish the regularities of the manifestation of valuable traits in samples of different geographical origin in different soil and climatic zones. In our case, it is a Steppe zone of Ukraine. It should be noted that in connection with global warming climate, the Steppe and left-bank forest-steppe of Ukraine appear more dry and unfavorable for the cultivation of peas, area and production of which in the last few years in these regions has decreased dramatically [1]. Some authors believe that in the steppe zone of Ukraine to revive the acreage under leguminous crops is possible only through the use of drought - and heat-resistant crops adapted for mechanized cultivation. This meets the requirements of culture chickpea [2]. According to its biological and morphological characteristics chickpeas (Cicer arietinum L.) - a typical xerophyte. Breeding programs for peas (Pisum sativum L.) in the steppe zone (zone of insufficient moisture) are aimed at obtaining crop drought-resistant varieties. The potential for legumes is not only not used adequately, but not yet fully defined [3].

\section{MATERIALS, METHODS AND CONDITIONS OF RESEARCH}

Sowing was carried out in the optimum for pea and chickpea time of hand planters on plots with an area of $1 \mathrm{~m}^{2}$ with a scheme of planting $15 \times 10 \mathrm{~cm}$. Unit of standards were placed after every 10 samples.

Weather conditions in the years of research 2015, 2016 and 2017 years were marked by significant variability in the phases of ontogenesis of pea and chickpea plants. Excessively high temperatures in 2015 and 2016 years and lack of rainfall during the "budding-flowering" caused a low level of yield of peas. In 2017, during the growing season, the optimal conditions for obtaining high yields were formed. To characterize the weather conditions of 2015-2017, Selyaninov's hydrothermal coefficient (SCC) was used, which in 2015 was 0.57 , in $2016-0.60$, in $2017-0.71$ (Fig.1). It is proved that the best conditions for obtaining high yields of leguminous crops in spring CIBS are created when the SCC for the corresponding period is 1-1.4. At a value of 0.6 and less plants are suppressed by drought, and at a value of 1.6 and more are suppressed by waterlogging [4]. It should be noted that 2015 and 2016 were distinguished by a low level of moisture supply during the growing season and a high temperature regime.

Objects of research-collection samples of peas and chickpea.

The morphological description of samples, their classification on economic and biological properties is carried out on classifiers of the corresponding sorts:

- Guidelines for the study of the collection of grain legumes [5];

- A wide unified classification of CMEA and the international classification of genus Pisum L. [6];

- The classifier of the genus Cicer L. [7];

- Wide unified classifier of genus Cicer L. [8].

Statistical processing of obtained data was performed according to the method of Rokitsky P. F. [9], analysis of variance - method Dospekhov B.A., John E. Freund [10, 11].

The results provide an opportunity to identify the most productive and most valuable samples.

After the study, seed samples are put in storage and transpose to maintain its viability and germination in 3-4 years.

\section{RESEARCH RESULT}

The results of studies of peas

The study of pea samples allowed to identify the most valuable genotypes for use in breeding programs. According to the results of a three-year cycle of study conducted in 2015-2017, the selected sources of valuable economic characteristics of peas, which will be further attracted to the collections:

- the complex of valuable economic features were allocated 8 samples: Lug 128/13, LDS01175, UKR; Lug 78/13, LDS01169, UKR; Lug 74/13, LDS01167, UKR; LNAU I/13, LDS01180, UKR; LNAU V/13, LDS01184, UKR; LNAU IV/13 LDS01183, UKR; Lug 118/13, LDS01186, UKR; Lug 76/13, LDS01168, 
UKR Lug 76/13, LDS01168, UKR. All these samples have such valuable properties as ceremonialist, suitability for mechanized harvesting, high yielding, disease resistance (Fusarium) and pests (pea weevil);

- according to the duration of the growing season of ultra-and precocious samples (that is, samples that would have a vegetation period of less than 60 days) was not. In addition, during the years of testing, the duration of the growing season had significant fluctuations, which depended on the weather conditions of growing peas. Vegetation period 61-70 days was allocated 17 samples: Lug 76/13, LDS01168, UKR; Lug 78/13, LDS01169, UKR; Lug 121/13, LDS01173, UKR; Lug 128/13, LDS01175, UKR; Lug 194/06, LDS01176, UKR; Lug 229/07, LDS01177, UKR; Lug 235/07, LDS01178, UKR; Lug 236/07, LDS01179, UKR; LNAU, I/13, LDS01180, UKR; LNAU II/13, LDS01181, UKR; LNAU III/13, LDS01182, UKR; LNAU IV/13 LDS01183, UKR; LNAU V/13, LDS01184, UKR; Lug 77/13, LDS01185, UKR; Lug 118/13, LDS01186, UKR; Lug 126/13, LDS01187, UKR; Dinga, LDS01188, DEU;

- by weight of 1000 grains (one of the component signs of plant productivity) - no samples were found;

- suitability for mechanized harvesting (high attachment of the lower layer of beans above soil level (over 45 cm) - 23 samples: Svetozar, LDS01162, RUS; Pearl, LDS01163, RUS; Krasnoyarsk emerald, LDS01165, RUS; LNAU I/13, LDS01180, UKR; LNAU II/13, LDS01181, UKR; LNAU III/13, LDS01182, UKR; LNAU IV/13 LDS01183, UKR; Lug 76/13, LDS01168, UKR; Lug 78/13, LDS01169, UKR; Lug 121/13, LDS01173, UKR; Lug 128/13, LDS01175, UKR; Lug 194/06, LDS01176, UKR; Lug 229/07, LDS01177, UKR; Lug 235/07, LDS01178, UKR; Lug 236/07, LDS01179, UKR; LNAU V/13, LDS01184, UKR; Lug 77/13, LDS01185, UKR; Lug 118/13, LDS01186, UKR; Lug 126/13, LDS01187, UKR; Dinga, LDS01188, DEU;

- high seed yield (\% to standard) 115 was only one sample: Lug 76/13, LDS01168, UKR.

Among samples analyzed, it is worth mentioning a sample of LNAU I/13, LDS01180, UKR, which has high technological and productive parameters (tall, suitable to mechanized harvest, weight of 1000 seeds - $215,5 \mathrm{~g}$, yield is $283,3 \mathrm{~g}$ per $1 \mathrm{~m}^{2}$, sample Lug $76 / 13$, LDS01168, UKR - sample suitable for mechanized harvesting, has a high yield per $1 \mathrm{~m}^{2}-318,9 \mathrm{~g}$ and sample Lug 118/13, LDS01186 - suitable for mechanized harvesting, the yield from $1 \mathrm{~m}^{2}-284,4 \mathrm{~g}$. Samples are resistant to diseases and pests.

The results of studies of chickpea

The study of chickpea samples was carried out according to the "guidelines of VIR for the study of leguminous crops". Morphological description, classification by economic and biological properties was carried out according to the "Classifier of the genus Cicer L.". To describe the sign of "Bush shape" used this gradation: 1 - creeping, 2 - wide, 3 - standing and 4 - compact.

Chickpea samples with high or increased parameters of manufacturability and productivity:

- the complex of valuable managemental and signs stood out 12 samples: Ikel, LDS00808, MDA; Odysseus, LDS00810, RUS; Treasure LDS00811, RUS; Lug 801/13, LDS00819, UKR; Lug 77/09, LDS00823, UKR; Lug 52/09, LDS00825, UKR; Lug 99/11, LDS00826, UKR; Lug 89/10, LDS00827, UKR; Lug 101/11, LDS00828, UKR; Lug 57/11, LDS00829, UKR; Lug 58/09, LDS00830, RUS; Lug 61/10, LDS00832, UKR;

- vegetation period 71-75 days 2 samples: Lug 38/13, LDS00813, UKR; Lug 51/13, LDS00815, UKR;

- duration of the vegetation period is 76-80 days - 22: Ikel, LDS00808, MDA; Botna, LDS00809, MDA; Odysseus, LDS00810, UKR; Treasure LDS00811, UKR; Lug 36/13, LDS00812, UKR; Lug 45/13, LDS00814, UKR; Lug 54/13, LDS00816, UKR; Lug 56/13, LDS00817, UKR; Lug 89/13, LDS00818, UKR; Lug 801/13, LDS00819, UKR; Lug 964/13, LDS00820, UKR; Jordan, LDS00821, ISR; Lug 27/09, LDS00822, UKR; Lug 77/09, LDS00823, UKR; Lug 55/09, LDS00824, UKR; Lug 52/09, LDS00825, UKR; Lug 99/11, LDS00826, UKR; Lug 89/10, LDS00827, UKR; Lug 101/11, LDS00828, UKR; Lug 57/11, LDS00829, UKR; Meadow 58/09, LDS00830, UKR; Lug 61/10, LDS00832, UKR;

- the weight of 1000 grains: were samples with large seeds (1000 seed weight 251-350 g) - 17: Ikel, LDS00808, MDA; Botna, LDS00809, MDA; Lug 38/13, LDS00813, UKR; Lug 51/13, LDS00815, UKR; Lug 56/13, LDS00817, UKR; Lug 89/13, LDS00818, UKR; Lug 801/13, LDS00819, UKR; Lug 964/13, LDS00820, UKR; Jordan, LDS00821, ISR; Lug 27/09, LDS00822, UKR; Lug 77/09, LDS00823, UKR; Lug 
55/09, LDS00824, UKR; Lug 52/09, LDS00825, UKR; Lug 89/10, LDS00827, UKR; Lug 57/11, LDS00829, UKR; Lug 58/09, LDS00830, UKR; Lug 61/10, LDS00832 and very large seeds (1000 seed weight $>350 \mathrm{~g})-6$ : Odysseus, LDS00810, UKR $\left(\mathrm{m}_{1000}=373,4 \mathrm{~g}\right)$; Treasure, LDS00811, UKR $\left(\mathrm{m}_{1000}=\right.$ 392,0 g); Lug 36/13, LDS00812, UKR (m1000 = amounted to 401,4 g); Lug 45/13, LDS00814, UKR (m 1000 = 370,2 g); Lug 99/11, LDS00826, UKR ( $\left.m_{1000}=352,1 \mathrm{~g}\right)$; Lug 101/11, LDS00828, UKR $\left(\mathrm{m}_{1000}=392,0 \mathrm{~g}\right)$;

- high-tech, that is suitable for mechanized harvest, was 24 sample: Founded LDS00808, MDA; Botna, LDS00809, MDA; Odysseus, LDS00810, UKR; Treasure LDS00811, UKR; Lug 36/13, LDS00812, UKR; Lug 45/13, LDS00814, UKR; Lug 54/13, LDS00816, UKR; Lug 56/13, LDS00817, UKR; Lug 89/13, LDS00818, UKR; Lug 801/13, LDS00819, UKR; Lug 964/13, LDS00820, UKR; Jordan, LDS00821, ISR; Lug 27/09, LDS00822, UKR; Lug 77/09, LDS00823, UKR; Lug 55/09, LDS00824, UKR; Lug 52/09, LDS00825, UKR; Lug 99/11, LDS00826, UKR; Lug 89/10, LDS00827, UKR; Lug 101/11, LDS00828, UKR; Lug 57/11, LDS00829, UKR; Lug 58/09, LDS00830, UKR; Lug 61/10, LDS00832, UKR; Lug 38/13, LDS00813, UKR; Lug 51/13, LDS00815, UKR;

- plant productivity - result sign, high seed yield (\% of standard) 115 had 12 samples: Founded LDS00808, MDA; Odysseus, LDS00810, RUS; Treasure LDS00811, UKR; Lug 801/13, LDS00819, UKR; Lug 77/09, LDS00823, UKR; Lug 52/09, LDS00825, UKR; Lug 99/11, LDS00826, UKR; Lug 89/10, LDS00827, UKR; Lug 101/11, LDS00828, UKR; Lug 57/11, LDS00829, UKR; Lug 58/09, LDS00830, UKR; Lug 61/10, LDS00832, UKR.

The complex of symptoms should be considered separately samples Odysseus, LDS00810, UKR; Treasure, LDS00811, UKR; Lug 89/11, Lug 99/11, LDS00826, UKR; Lug 101/11, LDS00828, UKR - all these samples are precocious, suitable for mechanized harvesting, very large seeds (very large (>350 g), high seed yield, standing uush shape.

\section{SUMMARY}

As a result of the analysis of pea and chickpea samples, the following samples were identified, which have high technological and productive parameters:

- pea is LNAU I/13, LDS01180, UKR, which has high technological and productive parameters (srednjoskolki, tall, suitable to mechanized harvest, weight of 1000 seeds $-215,5 \mathrm{~g}$, yield is $283.3 \mathrm{~g}$ per $1 \mathrm{~m}^{2}$, sample Lug 76/13, LDS01168, UKR - sample srednjoskolki suitable for mechanized harvesting, has a high yield per $1 \mathrm{~m}^{2}-318,9 \mathrm{~g}$ and sample Lug 118/13, LDS01186 -srednjoskolki suitable for mechanized harvesting, yield from $1 \mathrm{~m}^{2}-284,4 \mathrm{~g}$. Samples are resistant to diseases and pests.

- chickpea by complex of signs should provide examples of Odysseus, LDS00810, RUS; Treasure LDS00811, UKR; Lug 89/10, LDS00827, UKR, Lug 99/11, LDS00826, UKR; Lug 101/11, LDS00828, UKR - all these samples of early ripening, suitable for mechanical harvesting, the seeds are very large (very large (>350 g), high seed yield, upright shape of the shrub.

In General, the study of all samples of peas and chickpea showed a high variety of features in growing them in the Steppe zone of Ukraine. When creating a material with high-tech and productive parameters, it is possible to recommend all of the above mentioned samples, which combine valuable economic characteristics, that is, samples on a set of features.

\section{LITERATURE}

1. Chekalin N. M. Tishchenko V.M., Batashova M. (2008). Is.selection and genetics of individual cultures: studies. benefit. Poltava: FOP S. V. Govorov, 368 p. [in Ukrainian].

2. Skitsky V.Y. (2010). Analysis of a collection of chickpea for use in breeding for increased adaptability in cultivation. Plant genetic resources. No. 8. P. 40-45. [in Ukrainian].

3. Vishnyakova M.A. (2008). the Main directions of study of the collection of leguminous VIR at the present stage. Plant genetic resources. No. 6. P. 9-14. [in Russian]. 
4. Sokol T.V., Petrakova V.P. (2011). determination of the sources of pea resistance to pathogens and pests for breeding in the conditions of Eastern forest-steppe of Ukraine. Plant genetic resources. No. 9. P. 68-76. [in Ukrainian].

5. Korsakov N. You., Adamov A. P., Budanova V. S. et al. (1975). Guidelines for the study of the collection of grain legumes; compilers Leningrad: VIR, 59 p. [in Russian].

6. Wide unified classifier of COMECON and the international classification of CMEA of the genus Pisum L. Leningrad, 1981. 46 p. [in Russian].

7. The classifier is of the genus Cicer L. Leningrad, 1975. 13 p. [in Russian].

8. Wide unified classifier of the genus Cicer L. Kharkiv, 2012. 45 p. [in Ukrainian].

9. Rokitsky P. F. (1961). Bases of variation statistics for biologists. Minsk. publishing house Belgosuniversiteta. 223 p. [in Russian].

10. Dospekhov B. A. (1979). Technique of field experience. Moscow: Kolos. 416 p. [in Russian].

11. John E. (1988). Freund. Modern elementary statistics. Prentice-Hall, Inc. Upper Saddle River, NJ, USA, $574 \mathrm{p}$. 